\title{
Viscoelastic Properties of Poly(Methyl Methacrylates) Prepared by Anionic Polymerization
}

\author{
Toshiro Masuda, Keishi Kitagawa, ${ }^{*}$ and Shigeharu OnOgI \\ Department of Polymer Chemistry, Kyoto University, Kyoto, Japan.
}

(Received April 1, 1970)

\begin{abstract}
The viscoelastic properties of poly(methyl methacrylates) obtained by anionic polymerization were measured in the molten state by means of a concentric cylinder type rheometer over wide ranges of frequency and temperature. From the frequency dependence of the storage modulus $G^{\prime}$ and the loss modulus $G^{\prime \prime}$, the characteristic parameters in the terminal zone, such as zero-shear viscosity $\eta_{0}$ and steady-state compliance $J_{e}{ }^{0}$ were evaluated, and the dependence of these parameters on the molecular weight $M$ was discussed. $\eta_{0}$ and $J_{e}{ }^{0}$ for samples having molecular weights higher than the critical molecular weight $M_{C}$ were respectively proportional to $M^{4.4}$ and $M$. The quasi-equiliblium modulus $G_{e N}^{0}$ and the average molecular weight between entanglement coupling loci, $M_{e}$, were also evaluated. $M_{e}$ was found to increase gradually from 6760 to 13100 with increasing molecular weight. The ratio $M_{C} / M_{e}$ was not constant but varied from 6 to 2, being larger than the value of 2 obtained by previous authors, both theoretically and experimentally. The parameters for this material are discussed in connection with molecular structure, together with those for anionic polystyrene published in the previous paper.
\end{abstract}

KEY WORDS Viscoelasticity/Poly(Methyl Methacrylate) / Anionic Polymerization / Entanglement Coupling / Steady-State Compliance / Molecular Weight / Modified Rouse Theory / Polarity /

The viscoelastic behavior of polymer liquids, such as concentrated polymer solutions and melts, is largely determined by the mechanisms known as entanglement coupling ${ }^{1,2}$ or intermolecular interaction between polymer chains. As is well known, the effects of entanglement coupling and accordingly those of molecular weight and molecular weight distribution on the viscoelastic properties are found particularly in the terminal and rubbery zones.

In the terminal or flow zone, the rheological behavior of polymer liquids can be characterized by the zero-shear viscosity $\eta_{0}$ and the steadystate compliance $J_{e}{ }^{0}$, which are defined as follows;

$$
\begin{aligned}
\eta_{0} & =\lim _{\omega \rightarrow 0} \frac{G^{\prime \prime}}{\omega} \\
J_{e}^{0} & =\lim _{\omega \rightarrow 0} \frac{G^{\prime}}{\omega^{2} \eta_{0}{ }^{2}}
\end{aligned}
$$

where $G^{\prime}$ and $G^{\prime \prime}$ are respectively the storage

* Present address: Fuji Photo Film Co., Minamiashigara-cho, Ashigarakami-gun, Kanagawa, Japan. and loss moduli, and $\omega$ is the angular frequency.

In recent years, many investigators have concluded for several polymers that $\eta_{0}$ is proportional to $M_{w}{ }^{3.4}$, where $M_{w}$ is the weight-average molecular weight. This result holds regardless of molecular weight distribution, provided that $M_{w}$ is higher than the critical molecular weight $M_{C}$. The molecular weight dependence of $J_{e}^{0}$ appears rather complicated. Ninomiya, et al., reported that $J_{e}^{0}$ for poly(vinyl acetates) ${ }^{4}$ was proportional to molecular weight, supporting the prediction of the modified Rouse theory presented by Ferry, et al., ${ }^{5}$

$$
J_{e}^{0}=\frac{2}{5} \cdot \frac{M}{\rho R T}
$$

where $\rho, R$, and $T$ are respectively the density of polymer, gas constant and absolute temperature. However, some recent studies for a few polymers such as polystyrene, ${ }^{6-8}$ poly $(\alpha$-methylstyrene $)^{9,10}$ and polyisoprene ${ }^{11}$ show that $J_{e}^{0}$ is quite independent of, or at least much less dependent on, molecular weight than this equation 
would predict.

One of the most important parameters in the rubbery zone is the quasi-equiliblium modulus $G_{e N}^{0}$ which is closely related to the average molecular weight $M_{e}$ or chain length $Z_{e}=j M_{e}$ / $M_{0}$ between the entanglement coupling loci, where $j$ is the number of chain atoms per monomer unit, and $M_{0}$ the molecular weight of a monomer unit. From an analogy to the kinetic theory of rubber elasticity, the relation between $G_{e N}^{0}$ and $M_{e}$ can be given ${ }^{2}$ by

$$
G_{e N}^{0}=1 / J_{e N}^{0}=g_{N} \rho R T / M_{e}
$$

where $g_{N}$ is a front factor near unity and $J_{e N}^{\text {j }}$ is referred to as the entanglement compliance. Although it has been shown in several casesincluding polystyrene of very narrow distribution of molecular weight ${ }^{8}$ - that $M_{e}$ does not depend on molecular weight, if $M \geq M_{C}$, this has not been fully confirmed for various polymers in general.

In our earlier study, ${ }^{12}$ the viscoelastic properties of poly(methyl methacrylate) prepared by radical polymerization were measured, and the effect of molecular weight was mainly discussed. However, the samples employed had rather broad molecular weight distributions, and therefore some rheological parameters such as $J_{e}{ }^{0}$, $G_{e N}^{0}, J_{e N}^{0}$ and $M_{e}$ could not be determined accurately.

The present paper is principally aimed at a more accurate evaluation of various rheological parameters for poly(methyl methacrylates) having narrower distributions and detailed discussion of the effect of molecular weight on these parameters. The characteristic parameters studied were the rheological parameters $\eta_{0}, J_{e}^{0}, G_{e N}^{0}$, $J_{e N}^{0}, M_{e}$, as well as the shift factor $a_{T}$.

\section{EXPERIMENTAL}

\section{Preparation of Samples}

Pol(ymethyl methacrylate) (PMMA) samples of different molecular weight were prepared by anionic polymerization at low temperature $\left(-78^{\circ} \mathrm{C}\right)$ under pressures below $10^{-6} \mathrm{mmHg}$, using tetrahydrofuran as the solvent and sodium naphthalene as the initiator. Each polymer thus prepared had a rather broad distribution of molecular weight and therefore was fractionated in acetone- $n$-hexane mixtures. The fractions were purified in methanol and then dried in a vacuum oven.

\section{Characterization of the PMMA Samples}

The weight-average molecular weight $M_{w}$ of each fraction was determined from the intrinsic viscosity in acetone at $25^{\circ} \mathrm{C}$ by the following equation $^{13}$

$$
[\eta]=7.5 \times 10^{-5} M_{w}^{0.70}
$$

The number-average molecular weight $M_{n}$ was determined by osmotic pressure measurements using the High Speed Membrane Osmometer,

Table I. The weight- and number-average molecular weights and the ratio of these values for anionic PMMA

\begin{tabular}{cccc}
\hline Sample & $M_{w}$ & \multicolumn{1}{c}{$M_{\imath}$} & $M_{w} / M_{n}$ \\
\hline MF 2 & 342000 & 224000 & 1.53 \\
MF 3 & 270000 & 188000 & 1.44 \\
MF 5 & 197000 & 146000 & 1.35 \\
MF 6 & 158000 & 139000 & 1.14 \\
MF 7 & 116000 & 98000 & 1.18 \\
MF 8 & 96300 & 71800 & 1.34 \\
MF 9 & 63900 & 52800 & 1.21 \\
MF10 & 45200 & 37100 & 1.22 \\
MF11 & 35100 & 25300 & 1.39 \\
MF12 & 28400 & 18000 & 1.58 \\
MF13 & 11400 & 9120 & 1.25 \\
\hline
\end{tabular}

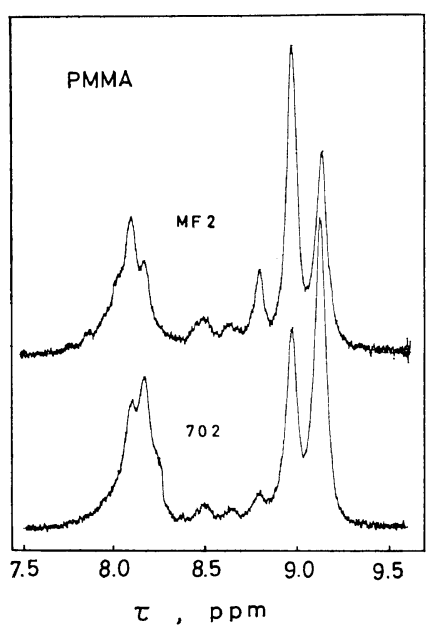

Figure 1. An Example of the NMR spectrum for anionic PMMA (MF2), compared with that for radical PMMA (702). 
Table II. The tacticity parameters for anionic and radical PMMA

\begin{tabular}{cccccccc}
\hline Samples & $M_{w}$ & $I$ & $H$ & $S$ & $i$ & $s$ & $B$ \\
\hline MF 2 & 342000 & 0.141 & 0.519 & 0.340 & 0.401 & 0.599 & 1.080 \\
MF 7 & 116000 & 0.141 & 0.512 & 0.347 & 0.397 & 0.603 & 1.063 \\
702 & 174000 & 0.065 & 0.370 & 0.565 & 0.250 & 0.750 & 0.987 \\
910 & 52000 & 0.063 & 0.406 & 0.531 & 0.266 & 0.734 & 1.040 \\
\hline
\end{tabular}

Model 502, of Mecrolab Inc. Values for $M_{w}$, $M_{n}$ and the ratio $M_{w} / M_{n}$ for the eleven samples used in this study are tabulated in Table I.

The tacticity of the samples prepared by anionic polymerization was examined by nuclear magnetic resonance (NMR) spectroscopy and compared with that of PMMA obtained by the radical polymerization. An example of the NMR spectrum for anionic PMMA is shown in Figure 1 together with that for radical PMMA. The tacticity parameters proposed by several authors, ${ }^{14-16}$ triad $(I, H$ and $S)$, diad ( $i$ and $s$ ) and heterogeneity parameter $B$ were evaluated from these spectra, and the results are shown in Table II. In this table, the samples MF 2 and MF 7 are the anionic PMMA used in this study, while 702 and 910 are the radical PMMA used in the previous study. ${ }^{12}$

The NMR spectra shown in Figure 1 and the tacticity parameters in Table II show that the samples of anionic PMMA are a little more isotactic than the radical PMMA. Furthermore, the amorphous character of the samples, as determined by the X-ray diffraction method, was found to be about the same in the anionic material as in the radical material.

\section{Measurements of Viscoelasticity}

The measurements of viscoelastic properties were carried out with the concentric cylinder type of rheometer, previously described. ${ }^{17}$ This rheometer enables us to measure the storage shear modulus $G^{\prime}$ and the loss modulus $G^{\prime \prime}$ as functions of the angular frequency $\omega$ at different temperatures. The measuring frequency ranged from $4 \times 10^{-3}$ to 0.5 , and the temperature from 120 to $280^{\circ} \mathrm{C}$.

The frequency dependence curves of the viscoelastic functions at various temperatures were superposed into their respective master curves by the use of the time-temperature superposition principle.

\section{RESULTS AND DISCUSSION}

Frequency Dependence of Viscoelastic Functions

In Figures 2 and 3 are shown, respectively, the master curves of the storage shear modulus $G^{\prime}$ and loss modulus $G^{\prime \prime}$ for the PMMA samples having different molecular weights. The abscissa in the figure is the reduced angular frequency $\omega a_{T}$, where $a_{T}$ is the shift factor determined from the time-temperature superposition, and the reference temperature is $220^{\circ} \mathrm{C}$. The mole-

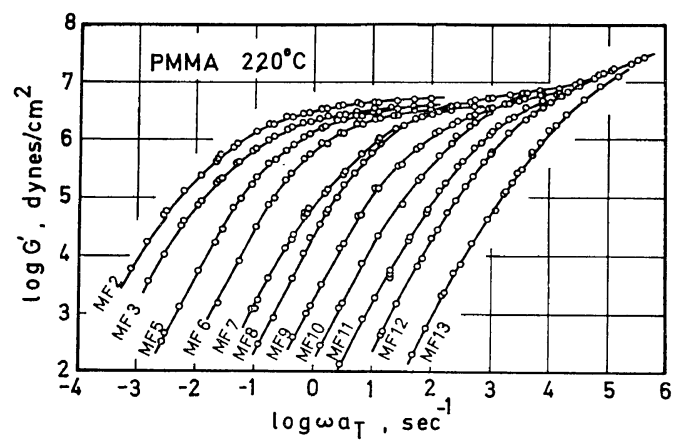

Figure 2. Master curves of $G^{\prime}$ for anionic PMMA having different molecular weights. The reference temperature is $220^{\circ} \mathrm{C}$.

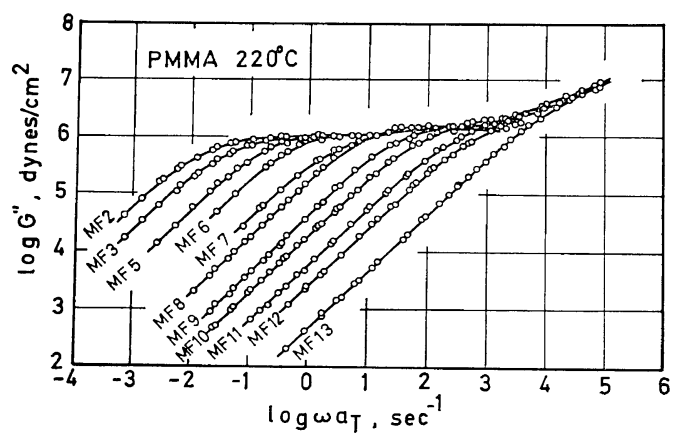

Figure 3. Master curves of $G^{\prime \prime}$ for anionic PMMA having different molecular weights. The reference temperature is $220^{\circ} \mathrm{C}$. 
cular weight of the samples ranges from 342000 to 11400 . As seen from these figures, the master curves include not only the terminal and rubbery zones but also the long time end of the glass transition zone. In the terminal zone the $G^{\prime}$ and $G^{\prime \prime}$ curves have, respectively, the slopes of 2.0 and 1.0, and shift to the lower frequency side and the rubbery plateau becomes longer and longer as the molecular weight increases.

All $G^{\prime}$ and $G^{\prime \prime}$ curves unite in the glass transition zone, excepting these for the sample MF13 having very low molecular weight. The $G^{\prime}$ curves for the samples having higher molecular weights manifest the rubbery plateau, whose intensity is about $3-6 \times 10^{6}$ dynes $/ \mathrm{cm}^{2}$.

\section{Shift Factor $a_{T}$-Effect of Temperature}

The shift factor $a_{T}$ for the PMMA samples used in this study is plotted against temperature $T$ in Figure 4. The large open circles in this

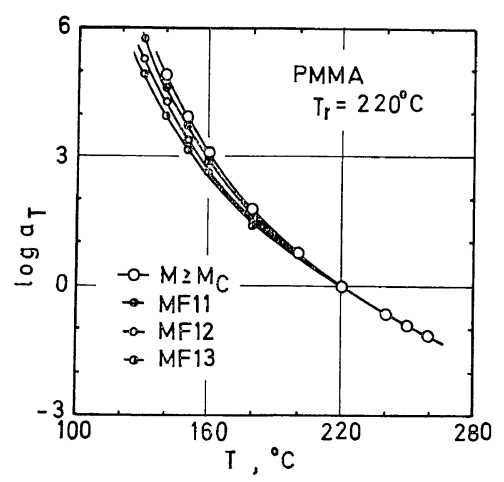

Figure 4. The logarithm of the shift factor $a_{T}$ plotted against temperature for anionic PMMA. Large open circles represent the results for $M \geq M_{C}$ and other circles for the samples indicated.

figure represent results for the samples having molecular weights of 45200 and higher, while the other circles are those for the samples indicated. The shift factor is independent of molecular weight provided that the molecular weight exceeds a certain limiting value. This limiting value, about 40000 , can be referred to as the critical molecular weight $M_{C}$ and will be disccussed in more detail later. For the polymers of lower molecular weight, the shift factor decreases with decreasing molecular weight, as has been reported for some other polymers. ${ }^{3}$
By the use of several parameters in the free volume theory, ${ }^{3}$ the WLF equation ${ }^{2,18}$ can be expressed as follows

$$
\begin{aligned}
& \log a_{T}=\frac{-c_{1}{ }^{g}\left(T-T_{g}\right)}{c_{2}{ }^{g}+\left(T-T_{g}\right)} \\
& \log a_{T}=\frac{-c_{1}^{r}\left(T-T_{r}\right)}{c_{2}^{r}+\left(T-T_{r}\right)}
\end{aligned}
$$

In these equations, $c_{1}^{g}=1 / 2.303 f_{g}, \quad c_{2}^{g}=f_{g} / \alpha_{f}$, $c_{1}{ }^{r}=1 / 2.303 f_{r}, c_{2}{ }^{r}=f_{r} / \alpha_{f}$, glass transition temperature $T_{g}$ and the reference temperature $T_{r}$, where $f_{g}$ and $f_{r}$ are respectively the fractional free volume at $T_{g}$ and $T_{r}, \alpha_{f}$ the temperature coefficient of the fractional free volume $f(T)$ defined by the following approximate equation above $T_{g}$

$$
f(T)=f_{g}+\alpha_{f}\left(T-T_{g}\right)=\alpha_{f}\left(T-T_{0}\right)
$$

$T_{r}$ was chosen as $220^{\circ} \mathrm{C}$ in this study and $T_{0}$ is well known as the Vogel temperature. Eq 7 shows that the plot of $-\left(T-T_{r}\right) / \log a_{T}$ against $\left(T-T_{r}\right)$ should give a straight line and that the parameters can be evaluated from the slope and the intercept. The plots for anionic PMMA are given in Figure 5. The free volume parameters calculated from this figure are tabulated in Table III, and $c_{1}{ }^{r}$ and $c_{2}^{r}$ are, respectively, 6.69 and 189.8 for the samples of high molecular weight. When $T_{g}$ is assumed to be $105^{\circ} \mathrm{C}$ for PMMA, ${ }^{19} c_{1}{ }^{g}$ and $c_{2}{ }^{g}$ can be calculated to be 16.98 and 74.76 , respectively, for the same samples.

As seen from Table III, $\alpha_{f}$ increases with decreasing molecular weight below $M_{C}$, while

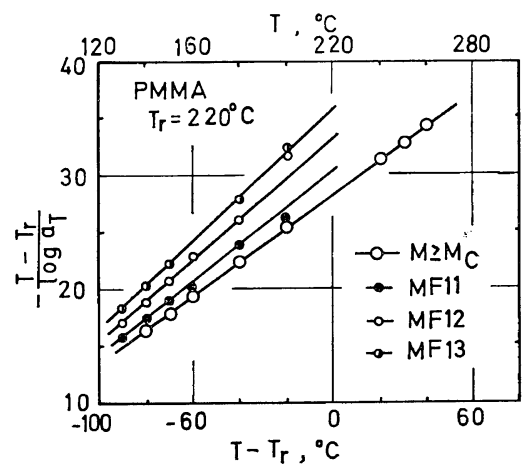

Figure 5. Linear plots of $-\left(T-T_{r}\right) / \log a_{T} v s$. $\left(T-T_{r}\right)$ for anionic PMMA. The circles are used as in Figure 4. 
Table III. Free volume parameters from the temperature dependence of the shift factor

\begin{tabular}{lrrrrrr}
\hline Sample & $M_{w}$ & $\alpha_{f}, \mathrm{deg}^{-1}$ & $T_{0},{ }^{\circ} \mathrm{C}$ & $f_{220}$ & $T_{r^{\prime}},{ }^{\circ} \mathrm{C}$ & $f_{g}$ \\
MF2-10 & $\geq 45200$ & $3.42 \times 10^{-4}$ & 30.2 & 0.065 & 220.0 & 0.026 \\
MF11 & 35100 & $3.96 \times 10^{-4}$ & 37.1 & 0.073 & 200.8 & $\left(T_{g}, 105^{\circ} \mathrm{C}\right)$ \\
MF12 & 28400 & $4.20 \times 10^{-4}$ & 34.4 & 0.078 & 189.1 & \\
MF13 & 11400 & $4.48 \times 10^{-4}$ & 34.4 & 0.083 & 178.9 & \\
\hline
\end{tabular}

$T_{0}$ seems to be unchanged. This is in marked contrast to the case of the anionic polystyrenes of narrow molecular weight distribution used in the previous study, ${ }^{8}$ where $\alpha_{f}$ was constant while $T_{0}$ decreased. The value of $f_{g}$ for the PMMA samples having molecular weights above $M_{C}$ is 0.026 , assuming $T_{g}=105^{\circ} \mathrm{C}$. This value compares well with the famous value of 0.025 .

The reference temperature $T_{r}{ }^{\prime}$ in Table III is the temperature at which the polymer having very low molecular weight has the same fractional free volume, namely, 0.065 as polymers having molecular weights above $M_{C}$ at $220^{\circ} \mathrm{C}$. At this temperature, each system has the same fractional free volume, and this state is often referred to as the iso-free-volume state..$^{2,18}$

\section{Rheological Behavior in the Terminal Zone}

The molecular weight dependence of the zeroshear viscosity $\eta_{0}$ for anionic PMMA at $220^{\circ} \mathrm{C}$ is shown by the open circles in Figure 6 . The

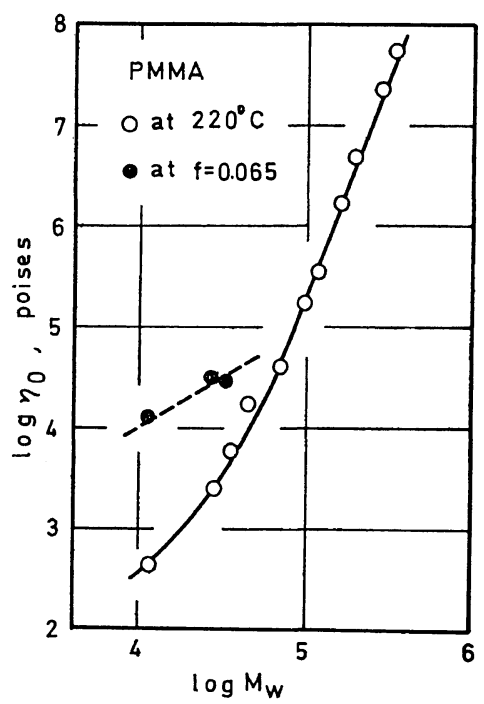

Figure 6. Molecular weight dependence of zeroshear viscosity $\eta_{0}$ for anionic PMMA at $220^{\circ} \mathrm{C}$ (open circles) and at $f=0.065$ (closed circles). dependence can be represented by a straight line having the slope of 4.4 in the highe1 molecular weight range. This slope is not only higher than the famous values of 3.4 but alsc higher than the value of 4.0 for the radical PMMA in the previous paper. ${ }^{12}$

The critical molecular weight $M_{C}$ for the anionic PMMA cannot accurately be determined from Figure 6, but it is likely to be located between 30000 and 40000 which is not too different from the reliable values of 27500 and 31500 , obtained by Fox, et al. ${ }^{3}$ The closed circles in Figure 6 represent the zero-shear viscosity at the temperature $T_{r}{ }^{\prime}$, or in the isofree-volume state where $f=0.065$. It is evident from Figure 6 that the molecular weight dependent of $\eta_{0}$ in the iso-free-volume state for the lower molecular weight PMMA can be represented by a straight line. It is also found that the molecular weight at the intersection of the two straight lines drawn in this figure is around 80000 , being much higher than the $M_{C}$ values so far published. This is in marked contrast to the case of the anionic polystyrenes ${ }^{8}$ studied previously, where $M$ at the intersection was very close to $M_{C}$. This discrepancy is associated with the fact that the dependence of shift factor on molecular weight is very great for PMMA as shown in Figures 4 or 5. The reason why such a peculiar result is obtained for PMMA is not clear, but it appears probable that the critical molecular weight above which the free volume does not vary with molecular weight it not always the same as $M_{C}$. The molecular weight at which the shift factor decreases may not be directly related to $M_{C}$ but rather only to the chemical structure of the polymer chains, because this high value of $M_{C}$ (80000) is not believed to be correct based upon the curve at $220^{\circ} \mathrm{C}$ shown in Figure 6 and certain other reliable values. ${ }^{3}$

In Figure 7 is shown the molecular weight 


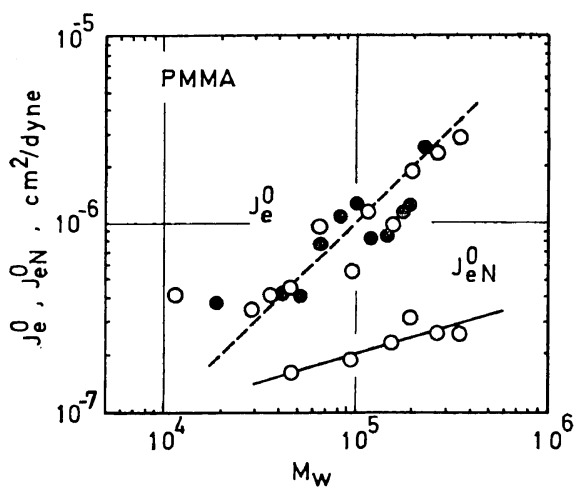

Figure 7. Molecular weight dependence of steadystate compliance $J_{e}{ }^{0}$ and entanglement compliance $J_{e N}^{0}$ for anionic PMMA (open circles) at $220^{\circ} \mathrm{C}$. Closed circles indicate the results for radical PMMA.

dependence of the steady-state compliance $J_{e}^{0}$, calculated by eq 2, for the anionic PMMA, together with that for the radical PMMA studied in the previous paper. ${ }^{12}$ It is clear from Figure 7 that $J_{e}{ }^{0}$ of both the radical and anionic PMMA is roughly proportional to the molecular weight. The broken line drawn in the figure shows the prediction of the modified Rouse theory ${ }^{5}$ given eq 3. The proportionality between $J_{e}{ }^{0}$ and $M$ has also been found for polyvinyl acetate (PVAc) by Ninomiya, et al.,${ }^{4}$ but not for several non-polar polymers, such as polystyrene and poly $(\alpha$-methylstyrene), as has been revealed by recent investigations. ${ }^{6-11}$ This discrepancy might be attributed to the polar groups in the polymer chains of PMMA and PVAc, though the effect of the branching in these materials should not be completely disregarded.

\section{Rheological Behavior in the Rubbery Zone}

As seen from Figure 2, all the $G^{\prime}$ curves do not show the flat rubbery plateau, and hence it difficult to determine the quasi-equilibrium modulus $G_{0 N}^{0}$ accurately. According to Sanders, et al. ${ }^{20,21}$ therefore, $G_{e N}^{0}$ was evaluated by the integration of $G^{\prime \prime}$ over values of $\ln \omega$ encompassing the maximum of $G^{\prime \prime}$

$$
G_{e N}^{0}=1 / J_{e N}^{0}=\frac{2}{\pi} \int_{-\infty}^{a^{\prime}} G^{\prime \prime} \mathrm{d} \ln \omega
$$

In practice, the $G^{\prime \prime} v s . \ln \omega$ curve was numerically integrated from $\ln \omega=-\infty$ to the maximum of $G^{\prime \prime}$ and the result was doubled. This
Table IV. Entanglement modulus and compliance, and entanglement spacings obtained by integration of the loss modulus

\begin{tabular}{lrccr}
\hline Sample & $M_{w}$ & $\begin{array}{c}G^{0}{ }_{e N}, \\
\mathrm{dyn} / \mathrm{cm}^{2}\end{array}$ & $\begin{array}{c}J^{0}{ }_{e N}, \\
\mathrm{~cm}^{2} / \mathrm{dyn}\end{array}$ & \multicolumn{1}{c}{$M_{e}$} \\
\hline MF 2 & 342000 & $3.94 \times 10^{6}$ & $2.54 \times 10^{-7}$ & 10400 \\
MF 3 & 270000 & $3.89 \times 10^{6}$ & $2.57 \times 10^{-7}$ & 10500 \\
MF 5 & 197000 & $3.12 \times 10^{6}$ & $3.20 \times 10^{-7}$ & 13100 \\
MF 6 & 158000 & $4.33 \times 10^{6}$ & $2.31 \times 10^{-7}$ & 9480 \\
MF 8 & 96300 & $5.41 \times 10^{6}$ & $1.85 \times 10^{-7}$ & 7580 \\
MF10 & 45200 & $6.07 \times 10^{6}$ & $1.65 \times 10^{-7}$ & 6760 \\
\hline
\end{tabular}

convenient procedure produces no serious error.$^{8,22}$ The calculated values of $G_{e N}^{0}$ and $J_{e N}^{0}$, and also the value of $M_{e}$ evaluated from eq 4 are tabulated in Table IV. It is clear from this table that $G_{e N}^{0}$ increases gradually, while $M_{e}$ decreases with decreasing molecular weight. $J_{e N}^{*}$, the reciprocal of $G_{e N}^{0}$, is logarithmically plotted against molecular weight in Figure 7 . The plot can be approximated by a straight line having the slope of 0.3 , as seen in the figure. This molecular weight dependence of $J_{e N}^{0}$ and $M_{e}$ in the entanglement region does not agree with the generally accepted viewpoint that $\partial \log$ $J_{e N}^{0} / \partial \log M=0$ nor with the result obtained by us for the anionic polystyrene. As seen from Table IV, $M_{e}$ ranges from 13100 to 6760 , while $M_{C}$ is estimated to be $30000-40000$. Therefore, the ratio $M_{C} / M_{e}$ is as high as $2-6$, though it is about 2 for the usual non-polar polymers. ${ }^{8}$ The anomaly found in the molecular weight dependence of $M_{e}$ and in the ratio $M_{C} / M_{e}$ for PMMA may be attributed to the fact that the polarity affects the internal structure, due to the entanglement couplings between molecular chains in the liquid.

Acknowledgement. This study was supported by a grant for scientific research (Kagaku Kenkyu-hi) from the Ministry of Education, Japan.

\section{REFERENCES}

1. J. D. Ferry, Proc. 5th Intern. Congress on Rheology, Vol. 1, S. Onogi, Ed., University of Tokyo Press, Tokyo, 1969, p 3.

2. J. D. Ferry, "Viscoelastic Properties of Polymers", 2nd ed, John Wiley and Sons, Inc., New York, N.Y., 1970.

3. G. C. Berry and T. G. Fox, Adv. Polym. Sci., 
5, 261 (1968).

4. K. Ninomiya and J. D. Ferry, J. Phys. Chem., 67, 2292 (1963).

5. J. D. Ferry, R. F. Landel, and M. L. Williams, J. Appl. Phys., 26, 359 (1955).

6. A. V. Tobolsky, J. J. Aklonis, and G. Akovali, J. Chem. Phys., 42, 723 (1965).

7. G. Akovali, J. Polym. Sci., Part A-2, 5, 875 (1967).

8. S. Onogi, T. Masuda, and K. Kitagawa, Macromolecules, 3, 109 (1970).

9. H. Odani, S. Kitamura, N. Nemoto, and M. Kurata, Rep. Prog. Polym. Phys. Japan, 10, 321 (1967).

10. T. Fujimoto, N. Ozaki, and M. Nagasawa, $J$. Polym. Sci., Part A-2, 6, 129 (1968).

11. N. Nemoto, private communication.

12. S. Onogi, T. Masuda, and T. Ibaragi, KolloidZ. Z. Polym., 222, 110 (1968).

13. G. V. Schulz, H.-J. Cantow, and G. Meyerhoff, J. Polym. Sci., 10, 79 (1953); F. W. Billmeyer,
Jr. and C. B. den Than, J. Amer. Chem. Soc., 77, 4763 (1955).

14. F. A. Bovey and G.V.D. Tiers, J. Polym. Sci., 44, 173 (1960).

15. G. Natta, J. Polym. Sci., 16, 143 (1955).

16. R. L. Miller and L. E. Nielsen, J. Polym. Sci., 46, 303 (1960).

17. M. Horio, S. Onogi, and S. Ogihara, J. Japan. Soc. Testing Mater., 10, 350 (1961).

18. M. L. Williams, R. F. Landel, and J. D. Ferry, J. Amer. Chem. Soc., 77, 3701 (1955).

19. W. A. Lee and G. J. Knight, "Polymer Handbook', J. Brandrup and E. H. Immergut, Ed., Interscience Publishers, New York, N.Y., 1966, p III-68.

20. J. F. Sanders, J. D. Ferry, and R. H. Valentine, J. Polym. Sci., Part A-2, 6, 967 (1969).

21. J. F. Sanders and J. D. Ferry, Macromolecules, 2, 440 (1969).

22. T. Masuda, K. Kitagawa, T. Inoue, and S. Onogi, Macromolecules, 3, 116 (1970). 